\title{
Vogt-Koyanagi-Harada syndrome, a rare association of Hodgkin's disease
}

\author{
S.Y. Chuah, A.J. Lyne' and M.W. Dronfield \\ Departments of Medicine and ${ }^{1}$ Ophthalmology, Peterborough District Hospital, Peterborough, \\ Cambridgeshire, $U K$
}

Summary: Vogt-Koyanagi-Harada syndrome (VKHS) is a well-documented clinical entity. We report the case of a 24 year old man who, within 5 months of the diagnosis of VKHS, developed Hodgkin's disease. Like VKHS, the aetiology of Hodgkin's disease is unknown. A viral factor has been suspected in the pathogenesis of both conditions. Similar immunological abnormalities have been described in both, and may be important predisposing factors.

\section{Introduction}

Vogt-Koyanagi-Harada syndrome (VKHS) is a well-documented clinical entity, mainly in Japanese literature. It was described by Vogt in $1906,{ }^{1}$ Harada in $1926^{2}$ and Koyanagi in $1914^{3}$ and 1929. ${ }^{4}$ Initially the Vogt-Koyanagi syndrome was considered as a separate entity from Harada's disease. The former consists of bilateral nontraumatic anterior uveitis, poliosis, vitiligo, alopecia and deafness. The latter is characterized by bilateral non-traumatic posterior uveitis and retinal detachment. In 1951, Cowper ${ }^{5}$ embraced the two diseases as a single clinical entity. The American Uveitis Society ${ }^{6}$ established the following diagnostic criteria: (1) no previous eye trauma; (2) posterior uveitis; (3) one or more of the following: bilateral iridocyclitis, papillitis, meningoencephalitis, pleocytosis of the cerebrospinal fluid, deafness, alopecia, poliosis and vitiligo.

Cipriani et al. ${ }^{7}$ reported a case of VKHS occurring in a patient who was treated for Hodgkin's disease 10 years previously. We report, here, a patient who, within 5 months of the diagnosis of VKHS, developed Hodgkin's disease, despite appropriate treatment of the former.

\section{Case report}

A 24 year old man was admitted with an 8-day history of headache, nausea and vomiting, and a 2-day history of deafness, dizziness and oscillopsia. On examination, he had bilateral acute red eyes

Correspondence: S.Y. Chuah, M.R.C.P., Leicester General Hospital, Leicester LE5 4PW, UK Accepted: 30 November 1990 with circum-corneal injection. He had bilateral fatiguable horizontal nystagmus. Fundoscopy revealed bilateral papilloedema with clouding of the media. Bilateral sensorineural deafness was present. Computerized tomography of his head was normal. Audiograms revealed profound hearing loss of 70 decibels bilaterally.

Five days after admission his visual acuity had deteriorated alarmingly to $6 / 60$ bilaterally. His brother noticed that his previously blue eyes had turned green. His pupils had become irregular, and detailed ophthalmic examination revealed bilateral anterior and posterior uveitis with papillitis, macular oedema and vitritis. Topical steroids and mydriatics were prescribed.

Lumbar puncture revealed no excess of white cells. Chest X-ray was normal. Toxoplasma and viral serology was negative, including herpes simplex, cytomegalovirus and human immunodeficiency virus. The diagnosis of Vogt-KoyanagiHarada syndrome was made and treatment started with intravenous hydrocortisone four times daily, changing after a few days to prednisolone orally $100 \mathrm{mg} /$ day. His condition improved rapidly and the dose of prednisolone was gradually reduced.

Two months after presentation, his visual acuity had improved to $6 / 5$ bilaterally. Four months after diagnosis, while on prednisolone $7.5 \mathrm{mg} / \mathrm{day}$, he presented with left supra-clavicular lymphadenopathy which was biopsied. The histological appearances were those of nodular sclerosing Hodgkin's disease (high grade, lymphocyte depleted subgroup). Chest radiographs showed a large left parahilar mass, with bilateral para-tracheal lymphadenopathy. Computerized tomography of his abdomen was normal. His disease was staged at $2 A$. In view of the unfavourable histology and the bulky disease he was treated with chemotherapy. 


\section{Discussion}

Vogt-Koyanagi-Harada syndrome (VKHS) is a clinical diagnosis. ${ }^{6}$ Cerebrospinal fluid pleocytosis strengthens the diagnosis but is not an absolute necessity. Our patient fulfilled the criteria laid down by the American Uveitis Society.

The aetiology of VKHS is unknown. It has been suspected to be a viral-induced ${ }^{8}$ autoimmune disease. ${ }^{9-11}$ The disorder affects tissues having a common embryological origin; the uvea, leptomeninges, the melanoblasts, ocular pigments and auditory labyrinth pigments all originate from the neural crest. Autoantibodies to melanin ${ }^{12,13}$ and myelin $^{14}$ have been described, accounting for the cutaneous and neurological manifestations respectively. Based on a surface marker, peripheral blood lymphocytes, which sometimes exhibit a suppressor function, were reduced in number in VKHS. ${ }^{15}$ VKHS has also been reported in association with ulcerative colitis, ${ }^{16}$ and with hypothyroidism and diabetes mellitus. ${ }^{17}$

Epstein-Barr virus ${ }^{18,19}$ and vaccination ${ }^{20}$ have been implicated in the pathogenesis of some cases of Hodgkin's disease. An imbalance between helper and suppressor immune activity (the latter being weak) has also been proposed as a predisposing factor in the development of Hodgkin's disease. $^{21}$ Hence there are immunological similarities between VKHS and Hodgkin's disease.

Hodgkin's disease has been shown to be the initiating cause of autoantibody production in immune thrombocytopenia, ${ }^{22-25}$ haemolytic anaemia $^{26}$ and autoimmune neutropenia. ${ }^{27,28}$ Insulin-receptor antibody has also been reported in Hodgkin's disease. ${ }^{29,30} \mathrm{It}$ is, therefore, possible that Hodgkin's disease could induce the production of autoantibodies to melanin and myelin too.

It is very unlikely that our patient had central nervous system invasion by Hodgkin's disease rather than VKHS. Central nervous system invasion is a very uncommon complication of Hodgkin's disease and is usually found only late in the illness. In a series of more than 2,000 patients, ${ }^{31}$ only 12 had central nervous system invasion and, indeed, only 34 cases have been reported in the last 60 years. $^{32,33}$ Our patient developed VKHS more than 4 months before the diagnosis of Hodgkin's disease.

\section{Acknowledgements}

We thank Dr Susan Lightman of Moorfields Eye Hospital for her invaluable advice.

\section{References}

1. Vogt, A. Frühzeitiges Ergrauen der Zilien und Bemerkungen über den sogenannten plötzlichen Eintritt dieser Veränderung. Klin Monatsschr Augenheilkd 1906, 44: 228-242.

2. Harada, Y. Clinical study of non-suppurative choroiditis. A report of acute diffuse choroiditis. Acta Soc Ophthalmol Jpn 1926, 30: 351-377.

3. Koyanagi, Y. Poliosis and alopecia associated with idiopathic uveitis. Acta Soc Ophthalmol Jpn 1914, 18: 1188-1192.

4. Koyanagi, Y. Dysakusis, Alopecia and Poliosis bei schwerer Uveitis incht traumatischen Ursprungs. Klin Monatsschr Augenheilkd 1929, 82: 194-211.

5. Cowper, A.R. Harada's disease and Vogt-Koyanagi syndrome uveoencephalitis. Arch Ophthalmol 1951, 45: 367-376.

6. Snyder, D.A. \& Tessler, H.H. Vogt-Koyanagi-Harada syndrome. Am J Ophthalmol 1980, 90: 69-75.

7. Cipriani, D., Landonio, G. \& Canepari, C. A case of Vogt-Koyanagi-Harada syndrome in a patient affected by Hodgkin's disease. J Neurol 1989, 236: 303-304.

8. Schlaegel, T.F. \& Morris, W.R. Virus-like inclusion bodies in subretinal fluid in uveoencephalitis. Am J Ophthalmol 1964, 58: $940-945$.

9. Sugiura, S. Vogt-Koyanagi-Harada disease. Jpn J Ophthalmol 1978, 22: 9-35.

10. Ohno, S. Immunological aspects of Behcet's and VogtKoyanagi-Harada disease. Trans Ophthalmol Soc UK 1981, 101: 335-341.

11. Lubin, J.R., Lowenstein, J.I. \& Frederick, A.R. Jr VogtKoyanagi-Harada syndrome with focal neurological signs. Am J Ophthalmol 1981, 91: 332-341.
12. Hammer, H. Cellular hypersensitivity to uveal pigment confirmed by leucocyte migration tests in sympathetic ophthalmia and the Vogt-Koyanagi-Harada syndrome. $B r J$ Ophthalmol 1974, 58: 773-776.

13. Matsuda, H. Electron microscopic studies on VogtKoyanagi-Harada syndrome and sympathetic ophthalmia with special reference to the melanocyte. Acta Soc Ophthalmol Jpn 1970, 74: 1107-1112.

14. Manor, R.S., Livini, E. \& Cohen, S. Cell-mediated immunity of human myelin basic protein in Vogt-Koyanagi-Harada syndrome. Invest Ophthalmol Vis Sci 1979, 18: 204-206.

15. Okubo, K., Kurimoto, S., Okubo, K., Matsumoto, K. \& Yokoyama, M.M. Surface markers of peripheral blood lymphocytes in Vogt-Koyanagi-Harada disease. J Clin Lab Immunol 1985, 17: 49-52.

16. Inversen, T.H. \& Sverrisson, T. Vogt-Koyanagi-Harada syndrome. A case report. Acta Ophthalmol 1986, 64: $235-238$.

17. Jaggarao, N., Voth, D. \& Jacobsen, J. The Vogt-KoyanagiHarada syndrome: association with hypothyroidism and diabetes mellitus. Postgrad Med J 1989, 65: 587-588.

18. McVay, J.R. Infection and Hodgkin's disease. Lancet 1981, ii: 589-590.

19. Kvale, G., Hoiby, E.A. \& Pedersen, E. Hodgkin's disease in patients with previous infectious mononucleosis. Int J Cancer 1979, 23: 593-597.

20. Bichel, J. Postvaccinal lymphadenitis developing into Hodgkin's disease. Acta Med Scand 1976, 199: 523-525.

21. Zwitter, M. Hodgkin's disease: 5 steps from autoimmunity to cancer. Med Hypotheses 1984, 15: 399-413. 
22. Hussein, K.K., Shaw, M.T. \& Oleinick, S.R. Autoimmune thrombocytopenia and peripheral neuropathy heralding Hodgkin's disease. South Med J 1975, 68: 1414-1416.

23. Khilani, P. \& Sarraf, M. The association of autoimmune thrombocytopenia and Hodgkin's disease. Oncology 1973, 28: $238-245$.

24. Berkman, A.W., Woog, J.J., Kickler, T.S. \& Ettinger, D.S. Serial determination of antiplatelet antibodies in a patient with Hodgkin's disease and autoimmune thrombocytopenia. Cancer 1983, 51: 2057-2060.

25. Kim, H.D. \& Boggs, D.R. A syndrome resembling idiopathic thrombocytopenic purpura in 10 patients with diverse forms of cancer. Am J Med 1979, 67: 371-377.

26. Eisner, E., Ley, A.B. \& Mayer, K. Coombs' positive hemolytic anemia in Hodgkin's disease. Ann Intern Med 1967, 66: 258-273.

27. Heyman, M.R. \& Walsh, T.J. Autoimmune neutropenia and Hodgkin's disease. Cancer 1987, 59: 1903-1905.

28. Hunter, J.D., Logue, G.L. \& Joyner, J.T. Autoimmune neutropenia in Hodgkin's disease. Arch Intern Med 1982, 142: 386-388.
29. Braund, W.J., Naylor, B.A., Williamson, D.H. et al. Autoimmunity to insulin receptor and hypoglycaemia in patient with Hodgkin's disease. Lancet 1987, i: 237-240.

30. Walters, E.G., Tavare, J.M., Denton, R.M. \& Walters, G. Hypoglycaemia due to an insulin-receptor antibody in Hodgkin's disease. Lancet 1987, i: 241-243.

31. Sapozink, M.D. \& Kaplan, H.S. Intra-cranial Hodgkin's disease. A report of 12 cases and review of the literature. Cancer 1983, 52: 1301-1307.

32. Cuttner, J., Meyer, R. \& Yu, P.H. Intracerebral involvement in Hodgkin's disease. A report of 6 cases and review of the literature. Cancer 1979, 43: 1497-1506.

33. Marshall, G., Roessmann, U. \& Van den Noort, S. Invasive Hodgkin's disease of brain. Report of 2 new cases and review of American and European literature with clinical-pathologic correlations. Cancer 1968, 22: 622-630. 